\title{
Inactivation of P2YR12 contributes to isoflurane-induced neuronal injury by altering TLR-4/BDNF/TNF- $\alpha$
}

\author{
Nenghong Sun ${ }^{1}$, Lichao Chu ${ }^{1}$, Lei Yuan ${ }^{2}$, Zongcai $Q i^{1}$ \\ ${ }^{1}$ Department of Anesthesiology, Weifang People's Hospital, Weifang, Shandong, China, ${ }^{2}$ Department of Operating Theatre, \\ Weifang Seventh People's Hospital, Weifang, Shandong, China
}

\begin{abstract}
The present investigation evaluated the effect of inhibiting the P2Y12 gene on anaesthetic-induced neuronal injury in a rat model. Neuronal injury was induced by exposing the animals for $6 \mathrm{~h}$ to $30 \%$ oxygen containing $0.75 \%$ isoflurane and $1.2 \mathrm{mg} / \mathrm{kg}$ prasugrel (a P2Y12 inhibitor) p.o. for 14 days. Cognitive function was determined by the Morris water maze, and the neurological severity score was determined. Enzyme-linked immunosorbent assay was used to estimate the level of oxidative stress and mediators of inflammation in brain tissues of isoflurane-induced neuronal injury rats. Apoptosis of neuronal cells was estimated by terminal deoxynucleotidyl transferase dUTP nick end labelling (TUNEL) and western blot assays. Real time-polymerase chain reaction was performed to estimate the expression levels of several proteins. The data revealed that inhibiting the P2Y12 gene ameliorated changes in the modified neurological severity score and cognitive function in neuronal injury rats. Moreover the levels of proinflammatory mediators, oxidative stress, and cyclic AMP, and the number of TUNEL-positive cells, decreased significantly $(p<0.01)$ in the prasugrel-treated group compared to the negative control group. In addition, apoptosis of neuronal cells decreased in the prasugrel-treated group, as it ameliorated expression of the PI3K, BCl-2, Bad, and Akt proteins in the isoflurane-induced neuronal injury rats. Expression of brain-derived neurotrophic factor (BDNF) and tropomyosin receptor kinase $B$ (TrkB) proteins was enhanced, whereas the Toll-like receptor-4 (TLR-4) and nuclear factor $\kappa B$ (NF-KB) proteins decreased in the brain tissues of the prasugrel-treated group compared to the negative control group of rats. These results suggest that inhibiting the P2YR12 gene protects against neuronal injury in isoflurane-induced neuronal injury rats. Inhibiting the P2YR12 gene ameliorated neuronal apoptosis by regulating the BDNF/TLR-4/TNF- $\alpha$ pathway.
\end{abstract}

Key words: P2Y12 gene, prasugrel, neuronal injury, isoflurane, TUNEL assay.

\section{Introduction}

Anaesthetics are used for inducing analgesia during medical procedures and surgeries [5]. Millions of children are exposed to anaesthesia each year for medical procedures [20]. Isoflurane is a commonly used anaesthetic to induce anaesthesia. Exposure to isoflurane can lead to injury to several organs and neuronal degeneration [14]. Neuronal degeneration due to isoflurane causes impaired memory and learning in rodents [24]. In most in vivo studies on this subject, animals were exposed to $0.75 \%$ isoflurane, which does not affect respiration, haemodynamics, brain perfusion or blood glucose level $[15,23]$. Thus, the present study investigated the mechanism underlying the neurotoxicity induced by

\section{Communicating author}

Zongcai Qi, Department of Anesthesiology, Weifang People's Hospital, Weifang, Shandong, China, 261000,

phone/fax: 0086-0536-8192179, e-mail: TCockerellashen@yahoo.com 
$0.75 \%$ isoflurane. Paediatric patients aged < 4 years who were exposed to isoflurane showed loss of cognitive function and impaired memory [7]. Exposure to isoflurane stimulates the inflammatory process in the brain. Inflammation is induced by upregulation of inflammatory mediators, such as nuclear factor $\kappa B$ (NF- $\kappa \mathrm{B})$, tumour necrosis factor $\alpha$ (TNF- $\alpha)$ and Tolllike receptor-4 (TLR-4) [13]. Moreover, the immune system is activated by TLR-4 during neuronal injury, and brain-derived neurotrophic factor (BDNF) is activated in neuronal tissue by enhanced expression of PI3K [4]. BDNF activates the p75 receptor, tropomyosin receptor kinase $B(\operatorname{TrkB})$ and receptors on the cell surface, which demonstrates its neuroprotective effect [12]. The Akt protein activates growth factors that control apoptosis in neuronal cells. Anaesthetics are toxic to neuronal cells due to their action in the extracellular regulated kinase $1 / 2$ (ERK1/2) and c-Jun N-terminal kinase (JNK) signalling pathways [18]. The Akt protein is involved in JNK signallinginduced apoptosis of neuronal cells.

Management of memory impairment and neuronal injury remains a challenge; thus, a new therapeutic approach is required. P2Y12 is a G protein-coupled receptor located on glial cells and platelets [8] Some studies have revealed that the P2Y12 protein has a role in neuronal injury and repair [2]. Inhibiting P2Y12 is beneficial in neurological conditions, such as multiple sclerosis and Alzheimer's and Parkinson's disease, which are characterised by substantial pro-inflammatory responses, including microglial activation and release of ATP [11]. The inflammatory response and migration of cells are regulated by the P2Y12 protein, which can activate interleukin (IL) [27]. Moreover, one study reported that a P2Y12 antagonist attenuated ischemic stroke-induced neuronal injury by regulating endothelial nitric oxide synthase and the activity of the ERK1/2 pathway [17]. Herein, the role of P2Y12 expression was evaluated in an isoflurane-induced neuronal injury rat model.

\section{Material and methods}

\section{Animals}

Sprague-Dawley rat pups (age, 7 days) were purchased from Dashuo Laboratory Animal Reproduction Centre (Chengdu, China). The pups were maintained on a $12 \mathrm{~h}$ light : $12 \mathrm{~h}$ dark cycle under standard conditions (humidity: $60 \pm 5 \%$, temperature: $24 \pm 3^{\circ} \mathrm{C}$ ) as per the guidelines of Association for the Assessment and Accreditation of Laboratory Animal Care International (AAALAC) for experimentation and animal use. All study protocols were approved by The Institutional Animal Care and Use Committee of Weifang People's Hospital, Shandong, China (IACUC/WPH/2017/12).

\section{Experiment}

The animals were separated into three groups: a normal group, a negative control group that received only isoflurane, and a prasugrel-treated group that received $1.2 \mathrm{mg} / \mathrm{kg}$ p.o. prasugrel for 14 days. All animals were exposed to 30\% oxygen containing $0.75 \%$ isoflurane for $6 \mathrm{~h}$ on P7 [16]. At the end of the treatment protocol, the rats were killed, and the brain was isolated from each animal for assessment of neuronal apoptosis and protein expression.

\section{Determination of behavioural changes}

The Morris water maze (MWM) was used to determine behavioural changes as per a previously reported study [21]. The water maze had a height and diameter of 40 and $100 \mathrm{~cm}$, respectively, and the platform depth was $15.5 \mathrm{~cm}$. Four quadrants were created in the apparatus with thread and one quadrant of the platform was placed such that it could not be visualised. Swimming behaviour was monitored continuously for 6 days. Escape latency was determined as an indicator of spatial memory and the effect of a P2Y12 antagonist in isoflurane-induced rats was also determined.

\section{Estimate of neurological function}

The modified neurological severity score (mNSS) was used to determine balance, touch, vision, abnormal behaviour, muscle mass, sensation and motion, as previously reported [19]. The mNSS was calculated on a 0-18 scale, where 0 indicated normal brain function and 18 indicated a severe deficit. Neurological function was determined on days 1, 3, 7 and 14 .

\section{Assessment of cyclic AMP}

The levels of cyclic AMP were determined by an enzyme-linked immunosorbent assay (ELISA) kit in the brain tissues of the isoflurane and prasugrel-treated groups, as per the manufacturer's instructions. The cAMP level was expressed as pmol/mg. 


\section{Terminal deoxynucleotidyl transferase dUTP nick end labelling (TUNEL) assay}

The TUNEL assay was performed to evaluate neuronal apoptosis according to the manufacturer's instructions (Promega Inc., Madison, WI, USA). All rats were killed by cervical dislocation; the brains were then isolated and the hippocampus was dissected. The hippocampal tissues were dehydrated and seeded in paraffin. A microtome was used to section the tissues ( $6 \mathrm{~mm}$ thickness) with a spacing of about $200 \mu \mathrm{m}$. The number of apoptotic cells was estimated using NIS-Elements BR processing and analysis software (Nikon, Tokyo, Japan).

\section{Biochemical parameters}

ELISA kits were used to determine the concentrations of inflammatory mediators, such as NF- $\kappa B$, IL-1 $\beta$ and TNF- $\alpha$ in the brain tissues of isoflurane-induced neuronal injury rats. Moreover, oxidative stress markers, such as malondialdehyde (MDA) and super oxide dismutase (SOD), were measured in brain tissues using ELISA kits.

\section{Real-time-polymerase chain reaction (RT-PCR) analysis}

The RNA was isolated from hippocampal tissues using TRIzol reagent. The RevertAid First Strand cDNA Synthesis Kit (Fermentas, Ontario, Canada) was used to reverse-transcribe RNA. The primers mentioned below were mixed with RT2 SYBR Green Master Mix (Superarray, Frederick, MD, USA) to determine the gene expression using Quantitative SYBR Green PCR assays.

\section{Western blot assay}

Brain tissues from the site of injury were separated out and the tissue was lysed in lysis buffer. The supernatant was separated by centrifuging the lysate for $5 \mathrm{~min}$ at 10,000 rpm. Sodium dodecyl sulphate-polyacrylamide gel electrophoresis was used to separate the total proteins. The separated proteins were filtered onto a nitrocellulose membrane. The membrane was incubated with $I^{\text {ry }}$ antibodies, including PI3K (sc-136298; Santa Cruz Biotechnology, Santa Cruz CA, USA), Akt (sc-24500; Santa Cruz), Bcl-2 (sc-509; Santa Cruz), Bad (sc-8044; Santa Cruz), ERK-1/2 (sc-81492; Santa Cruz), NF-кB (sc-398442; Santa Cruz), TLR-4 (ab13556; Abcam, Cambridge, UK), JNK (ab47337;
Abcam), GADPH (sc-59540; Santa Cruz) and $\beta$-actin (ab8224; Abcam), overnight at $4^{\circ} \mathrm{C}$. Horseradish peroxidase-conjugated secondary antibody was incubated for $60 \mathrm{~min}$ at room temperature. Image J software (National Institutes of Health, Bethesda, MD, USA) was used to estimate band density.

\section{Statistical analysis}

All data are expressed as means \pm SEM $(n=10)$. One-way analysis of variance was performed, followed by Dunnett's post hoc test, using GraphPad Prism software (ver. 6.1.; GraphPad Software Inc., San Diego, CA, USA). The level of statistical significance was set at $p<0.05$.

\section{Results \\ Inhibiting P2Y12 improves cognitive function of isoflurane-induced neuronal injury rats}

The memory and cognitive function of the isoflurane-induced neuronal injury and prasugrel-treated group was estimated by the MWM test (Fig. 1). There was a significant $(p<0.01)$ reduction in the number of crossings and time spent in the target quadrant, as well as an increase in escape latency, in the negative control group compared to the normal group. However, prasugrel attenuated cognitive function deficits, shown by a greater number of crossings and time spent in the target quadrant, as well as reducing escape latency, in isoflurane-induced neuronal injury rats compared to the negative control group.

\section{Inhibiting P2Y12 reduces the mNSS of isoflurane-induced neuronal injury rats}

Rats treated with prasugrel and isoflurane were evaluated using the mNSS (Fig. 2). The mNSS was enhanced significantly in the negative control group compared to the normal group. There was a significant $(p<0.01)$ reduction in the mNSS in the prasugrel-treated group compared to the negative control group of rats.

\section{Inhibiting P2Y12 enhances cyclic AMP levels of isoflurane-induced neuronal injury rats}

The effect of inhibiting the P2Y12 gene with prasugrel on the level of cyclic AMP was examined in isoflurane-induced neuronal injury rats (Fig. 3). 

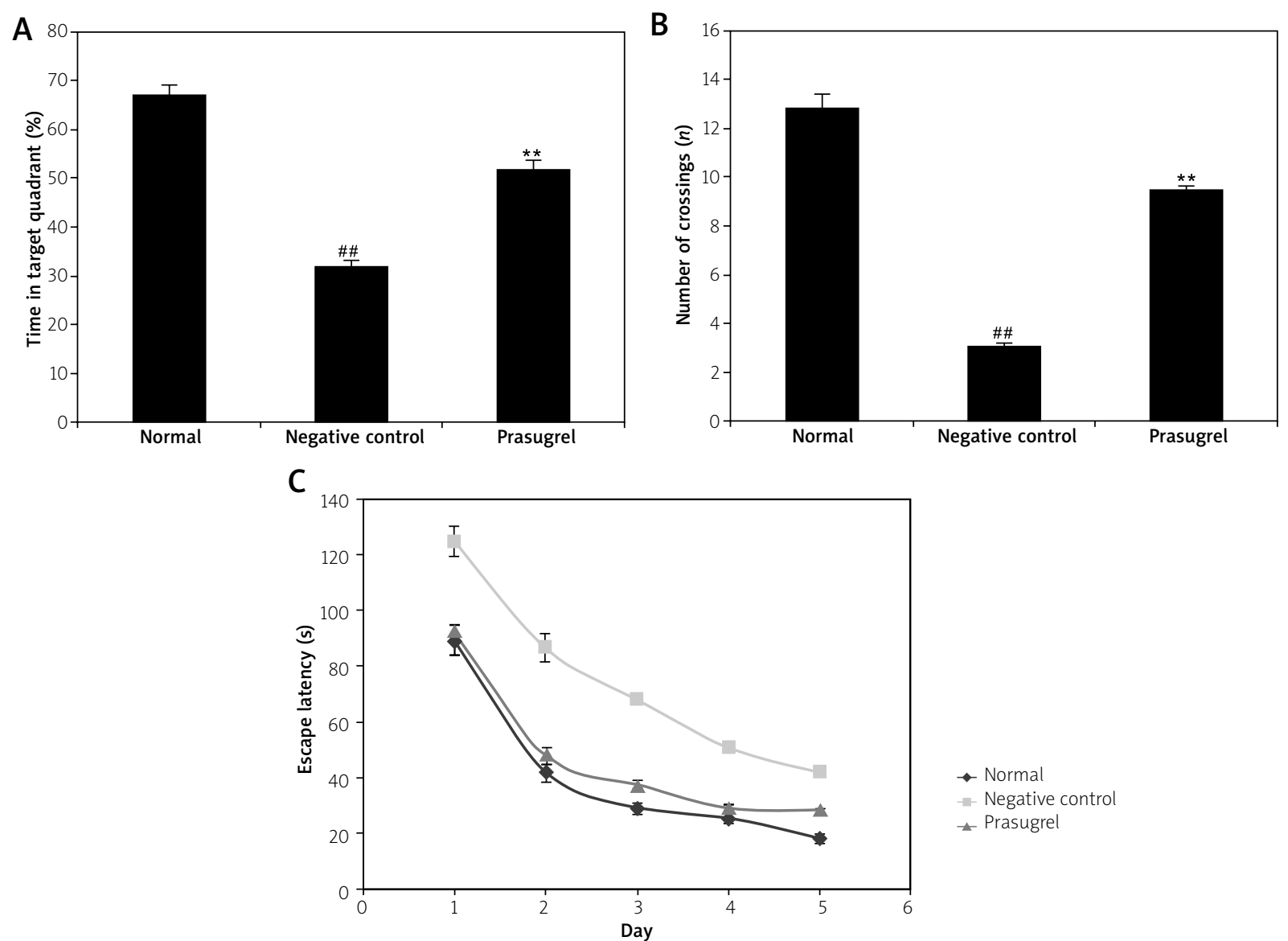

Fig. 1. Effect of a P2Y12 antagonist on cognitive function and memory (number of crossings, time spent in the target quadrant and escape latency) of isoflurane-induced neuronal injury rats: results of the Morris water maze test. Mean \pm standard error mean $(n=10) .{ }^{\# \#} p<0.01$ vs. normal group, ${ }^{* \star} p<0.01$ vs. negative control group.

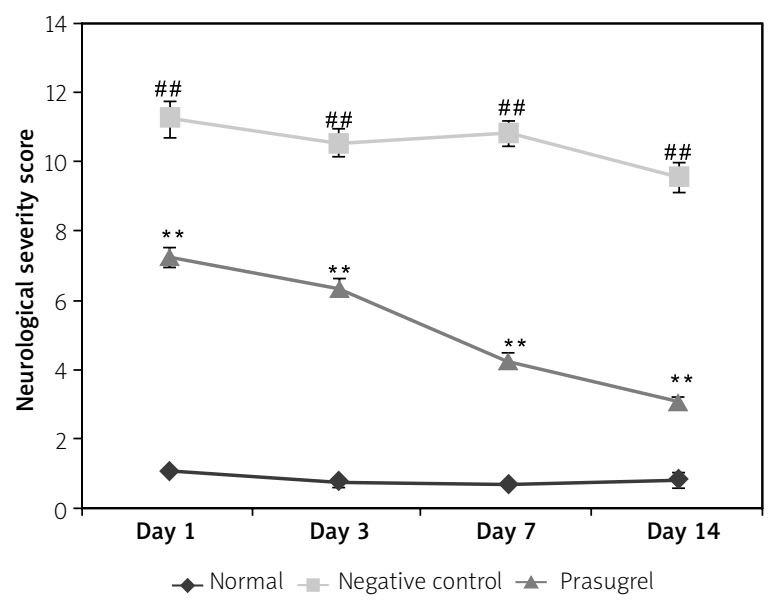

Fig. 2. Inhibiting the P2Y12 gene improves neurological function in isoflurane-induced neuronal injury rats, shown by lower modified neurological severity score (mNSS). Mean \pm standard error mean $(n=10) .{ }^{\# \#} p<0.01$ vs. normal group, ${ }^{* *} p<0.01$ vs. negative control group.

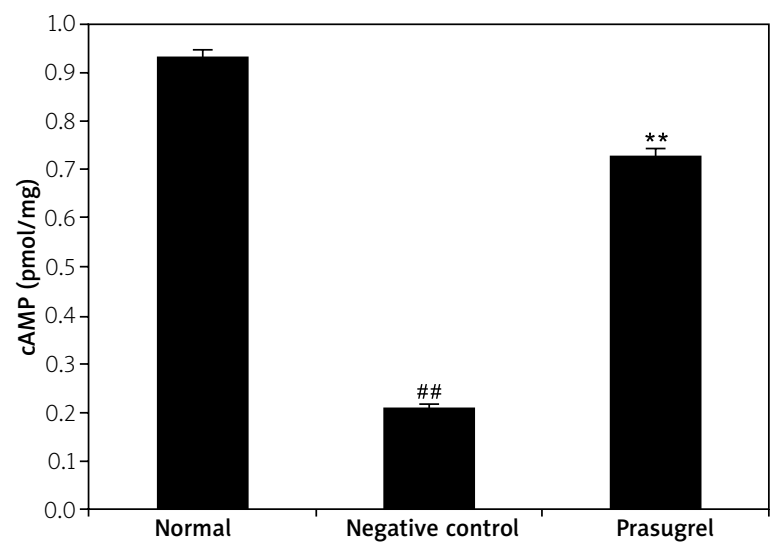

Fig. 3. Inhibiting the P2Y12 gene increases the level of cyclic AMP in brain tissues of isoflurane-induced neuronal injury rats. Mean \pm standard error mean $(n=10)$. ${ }^{\# \#} p<0.01$ vs. normal group, ${ }^{* *} p<0.01$ vs. negative control group. 
The level of cyclic AMP decreased significantly $(p<0.01)$ in the negative control group compared to the normal group. Prasugrel-induced inhibition of the P2Y12 gene effectively enhanced the level of cyclic AMP compared to the negative control group.

\section{Inhibiting $\mathrm{P} 2 \mathrm{Y} 12$ reduces the number of TUNEL-positive cells in isoflurane- induced neuronal injury rats}

The effect of inhibiting the P2Y12 gene on neuronal degeneration in isoflurane-induced neuronal injury rats was determined by the number of TUNEL-positive cells. The number of TUNEL-positive cells was significantly $(p<0.01)$ enhanced in the brain tissues of the negative control group compared to the normal group of rats. However prasugrel inhibited the P2Y12 gene and reduced the number of TUNEL-positive cells significantly $(p<0.01)$ compared to the negative control group (Fig. 4).
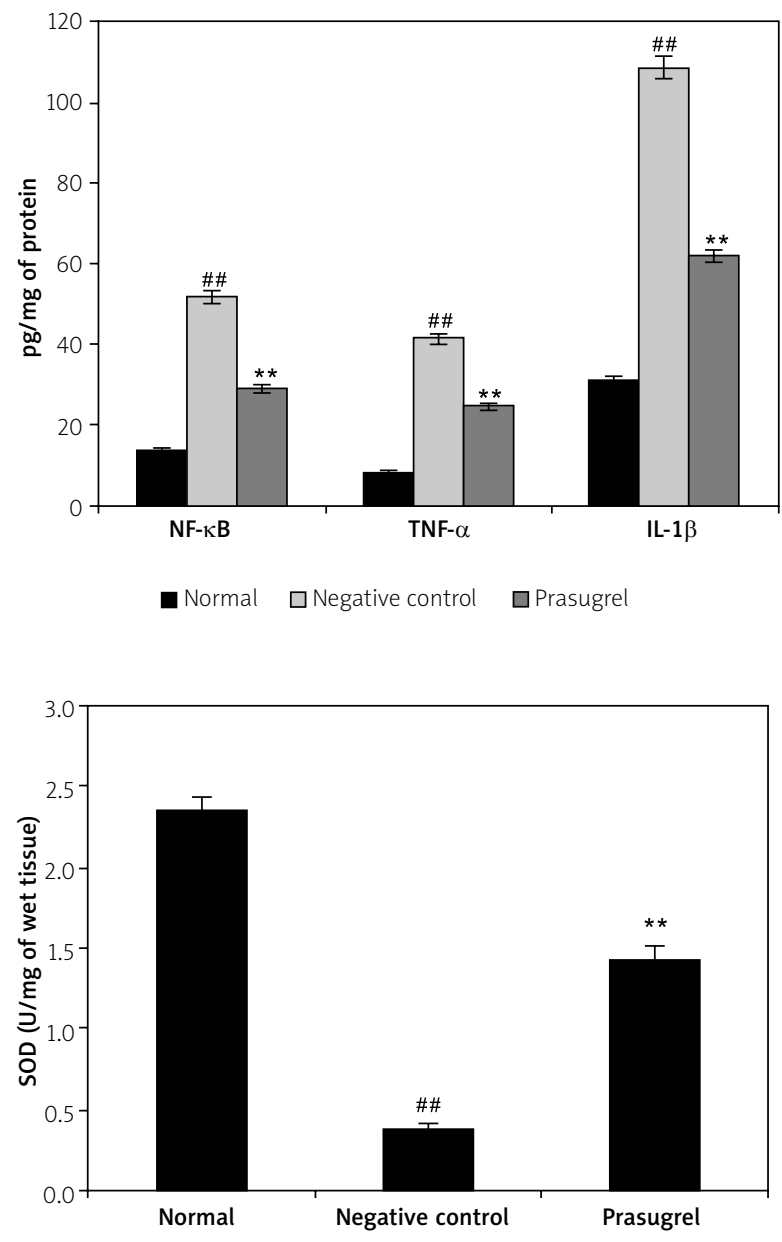

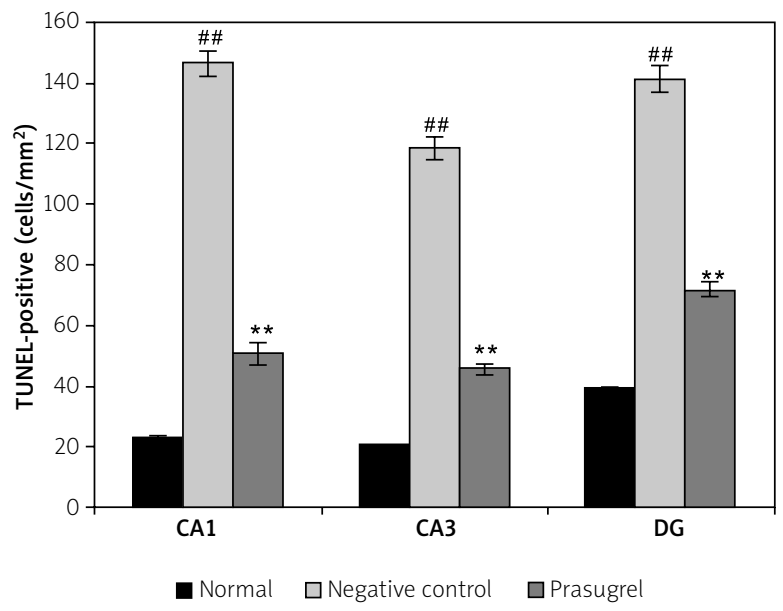

Fig. 4. Inhibiting the P2Y12 gene reduces the number of terminal deoxynucleotidyl transferase dUTP nick end labelling (TUNEL)-positive cells in brain tissues of isoflurane-induced neuronal injury rats. Mean \pm standard error mean $(n=10) .{ }^{\# \#} p<0.01$ vs. normal group, ${ }^{\star \star} p<0.01$ vs. negative control group.

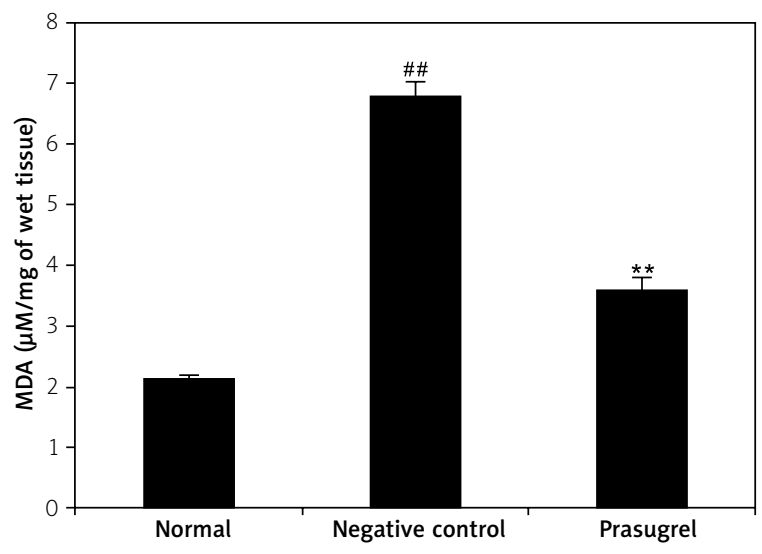

Fig. 5. Inhibiting the P2Y12 gene with prasugrel reduced the levels of oxidative stress and proinflammatory mediators in the brain tissues of isoflurane-induced neuronal injury rats. Mean \pm standard error mean $(n=10)$. ${ }^{\# \#} p<0.01$ vs. normal group, ${ }^{* *} p<0.01$ vs. negative control group. 


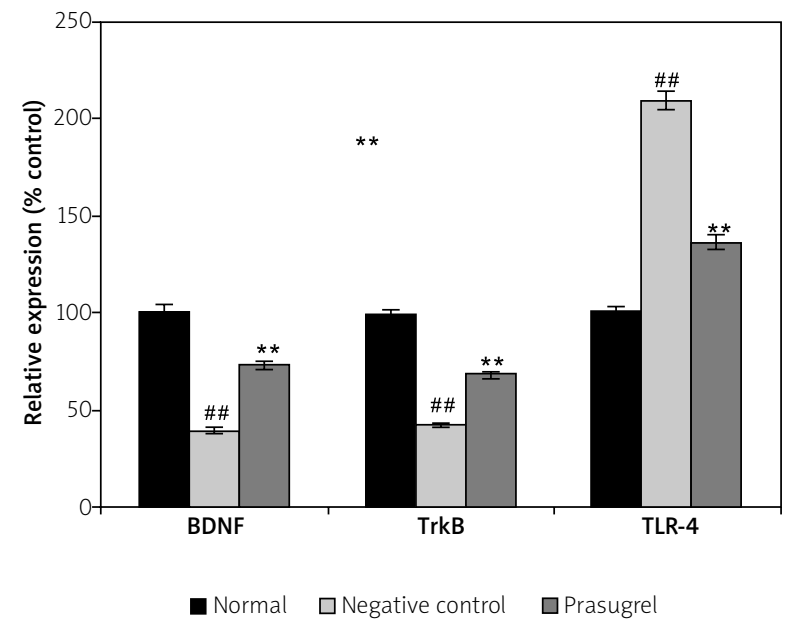

Fig. 6. Inhibiting the P2Y12 gene with prasugrel ameliorates gene expression of brain-derived neurotrophic factor (BDNF), tropomyosin receptor kinase B (TrkB) and Toll-like receptor-4 (TLR-4) in the brain tissues of isoflurane-induced neuronal injury rats, as shown by real-time-polymerase chain reaction (RT-PCR). Mean \pm standard error mean $(n=10) .{ }^{\# \#} p<0.01$ vs. normal group, ${ }^{* *} p<0.01$ vs. negative control group.

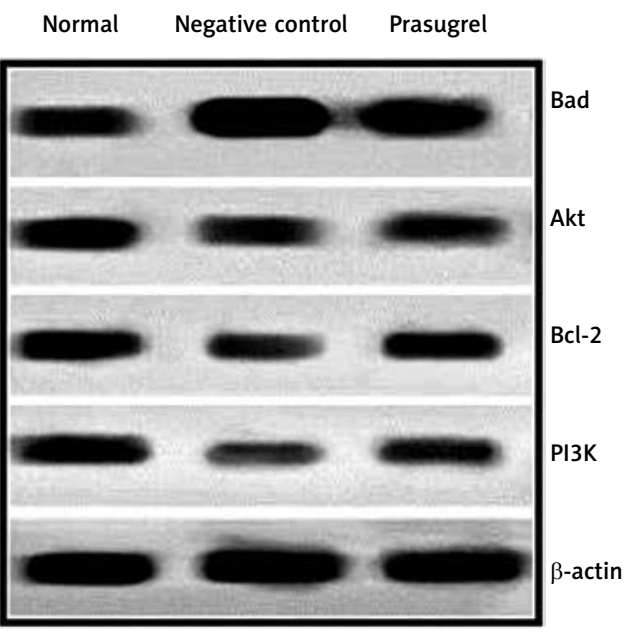

\section{Inhibiting P2Y12 reduces the levels of oxidative stress and inflammatory mediators in isoflurane-induced neuronal injury rats}

Isoflurane-induced neuronal injury rats treated with prasugrel showed altered levels of oxidative stress markers, such as MDA and SOD, and the production of proinflammatory mediators, such as $N F-\kappa B, T N F-\alpha$ and IL-1 $\beta$ (Fig. 5). The levels of NF- $\kappa B$, TNF- $\alpha$ and IL-1 $\beta$ were enhanced $(p<0.01)$ significantly in the brain tissues of the negative control group compared to the normal group of rats. Moreover, the level of oxidative stress was enhanced in the negative control group: the level of MDA increased and SOD activity was lower versus the normal group. Prasugrel reduced the levels of oxidative stress and inflammatory mediators significantly $(p<0.01)$ in the brain tissues of isoflurane-induced neuronal injury rats.

\section{Inhibiting P2Y12 ameliorates BDNF, TrKB and TLR-4 gene expression in isoflurane-induced neuronal injury rats}

The effects of prasugrel on the expression levels of the BDNF, TrKB and TLR-4 proteins in the brain tissues of isoflurane-induced neuronal injury rats are shown in Figure 6. A significant $(p<0.01)$ decrease in the relative expression of BDNF and TrKB, as well

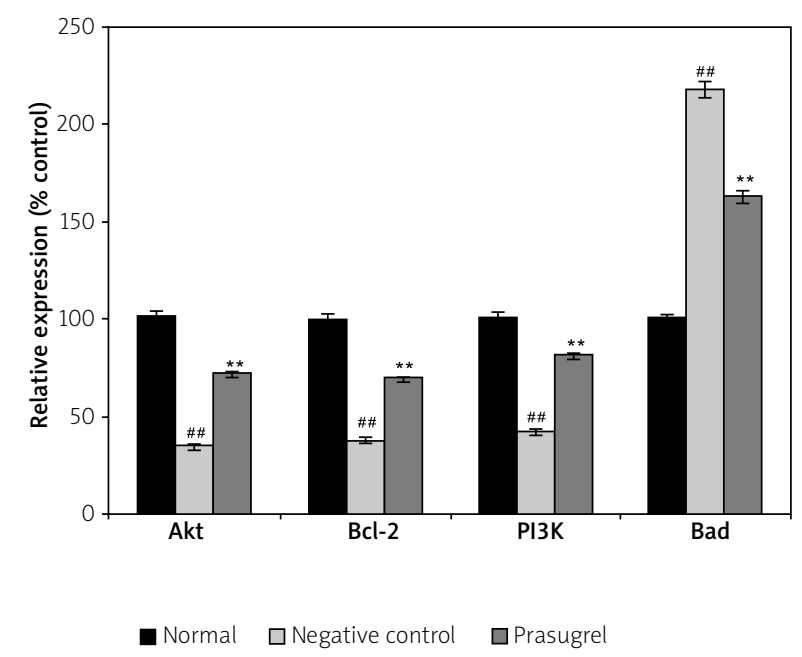

Fig. 7. Inhibiting the P2Y12 gene reduces neuronal apoptosis by attenuating the expression of Akt, Bcl-2, $\mathrm{PI} 3 \mathrm{~K}$ and the Bad protein in the brain tissues of isoflurane-induced neuronal injury rats, as shown by western blot assay. Mean \pm standard error mean $(n=10)$. ${ }^{\# \#} p<0.01$ vs. normal group, ${ }^{* *} p<0.01$ vs. negative control group. 

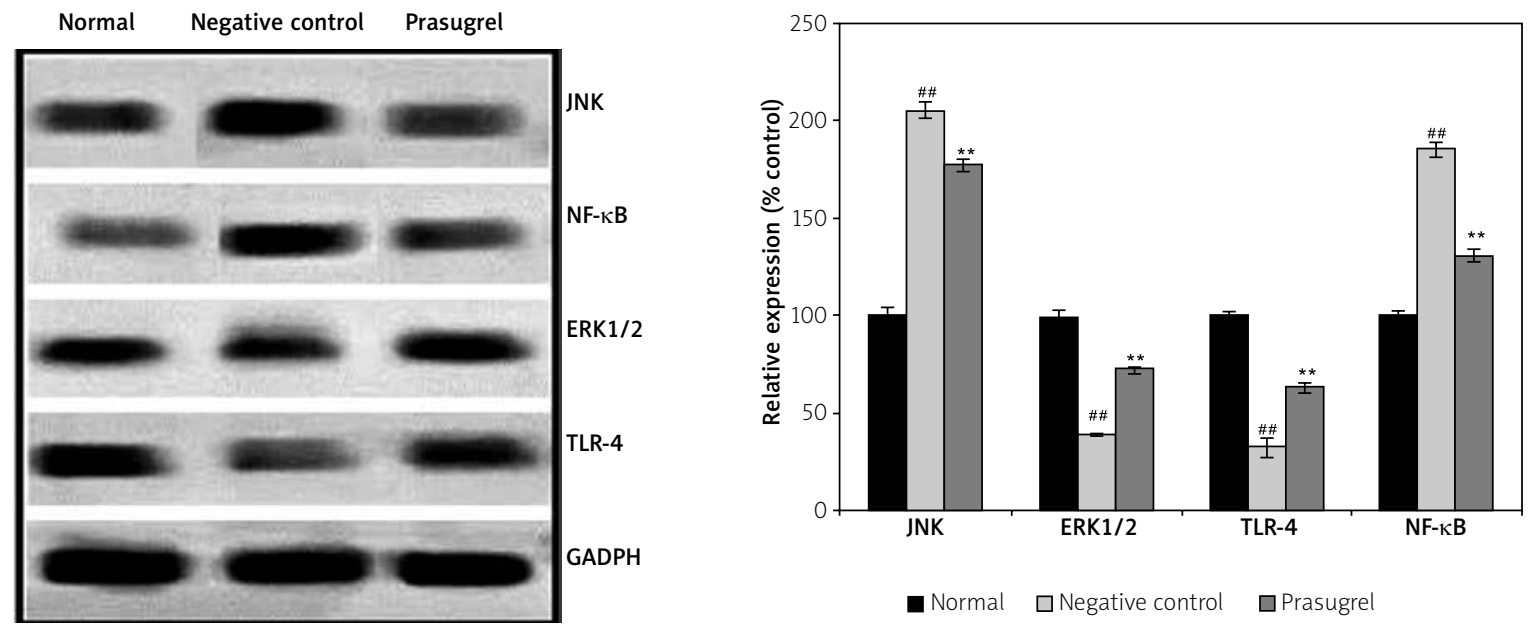

Fig. 8. Inhibiting the P2Y12 gene ameliorates the expression of ERK1/2, NF- $\mathrm{B} B$, TLR-4 and JNK proteins in brain tissues of isoflurane-induced neuronal injury rats, as shown by western blot. Mean \pm standard error mean $(n=10) .{ }^{\# \#} p<0.01$ vs. normal group; ${ }^{* *} p<0.01$ vs. negative control group.

as an increase in expression of TLR-4, were observed in the brain tissues of isoflurane-induced neuronal injury rats. However, inhibiting the P2Y12 gene significantly $(p<0.01)$ reduced the expression of the BDNF, TrKB and TLR-4 proteins in the brain tissues of isoflurane-induced neuronal injury rats.

\section{Inhibiting P2Y12 ameliorates neuronal apoptosis in isoflurane-induced neuronal injury rats}

Apoptosis of neurons was observed in the brains of the isoflurane- and prasugrel-treated neuronal injury rats by western blot assay (Fig. 7). Neuronal apoptosis was estimated by determining the gene expression of the $\mathrm{PI} 3 \mathrm{~K}, \mathrm{BCl}-2$, Bad and Akt proteins in the brain tissues of isoflurane-induced neuronal injury rats. The relative expression levels of the PI3K, $\mathrm{Bcl}-2$, Akt and Bad proteins decreased $(p<0.01)$ significantly in the negative control group compared to the normal group. However, prasugrel reduced the altered expression of the PI3K, Bcl-2, Bad and Akt proteins significantly $(p<0.01)$ in isoflurane-induced neuronal injury rats.

\section{Inhibiting P2Y12 ameliorates ERK-1/2, NF- $K B, T L R-4$ and JNK expression in isoflurane-induced neuronal injury rats}

The expression levels of the ERK-1/2, NF- $\kappa B$, TLR-4 and JNK proteins were determined in the prasu- grel-treated rats (Fig. 8). Enhanced expression of the $\mathrm{NF}-\mathrm{KB}$ and JNK proteins, and reduced expression of the ERK $1 / 2$ and TLR-4 proteins, were observed in the brain tissues of the negative control group compared to the normal group of rats. NF-KB and JNK expression decreased, and ERK-1/2 and TLR-4 protein expression significantly $(p<0.01)$ increased, in the prasugrel-treated group compared to the negative control group.

\section{Discussion}

Many medical procedures require anaesthesia, and many children are exposed to anaesthetics for medical procedures every year. Anaesthetics induce neuronal apoptosis, which leads to neuronal degeneration [6]. Furthermore, children exposed to anaesthetics develop memory impairments and cognitive dysfunction, and conventional treatments have many limitations. Thus, the present study determined the effect of inhibiting the P2Y12 gene on anaesthetic-induced neuronal injury in a rat model. Cognitive function was determined by the MWM and the mNSS. ELISA was used to determine the levels of oxidative stress markers and inflammatory mediators in the brain tissues of isoflurane-induced neuronal injury rats. Apoptosis of neuronal cells was estimated by the TUNEL and western blot assays, and RT-PCR was performed to estimate the levels of several proteins. 
The MWM provides a measure of spatial memory and cognitive function [3] and Behavioural changes can be determined with the mNSS [19]. The results of this study revealed that inhibiting the P2Y12 gene attenuated memory impairment and cognitive dysfunction, and also reduced the mNSS, in isoflurane-induced neuronal injury rats.

It has been well-documented that isoflurane alters the balance between anti- and pro-inflammatory cytokines in the brain [22]. Pro-inflammatory mediators, such as NF- $\mathrm{kB}$, TNF- $\alpha$ and IL-1 $\beta$, cause tissue damage due to aggregation of granulocytes [9]. Moreover, several reports have suggested that some drugs are beneficial against neuronal damage by reducing oxidative stress [25]. Activation of the P2Y12 receptor is neurotoxic due to the subsequent pro-inflammatory response, stimulation of phagocytosis, and migration of immune cells [11]. The present study revealed that inhibiting the P2Y12 gene reduced the levels of oxidative stress and proinflammatory mediators in isoflurane-induced neuronal injury rats.

One study reported that isoflurane induces apoptosis by altering the expression of proteins, such as Bcl-2, Bax and Bad, which are involved in controlling the apoptotic pathway [28]. These proteins maintain cell membrane integrity and release the factors involved in apoptosis. Moreover, the ERK and JNK pathways are involved in the regulation of the Bcl-2 protein [26]. Stimulating the P2Y12 gene leads to activation of inflammatory mediators and the Akt protein, which contributes to apoptosis [8]. The present data suggest that inhibiting the P2Y12 gene attenuated the altered expression of Bcl-2, Akt, Bad, JNK and ERK proteins in the brain tissues of anaesthetic-induced neuronal injury rats.

In addition, cell survival is regulated by the $\mathrm{PI} 3 \mathrm{~K} / \mathrm{Akt}$ pathway, as this pathway activates the anti-apoptotic proteins that inhibit apoptosis [10]. Our results suggest that inhibiting the P2Y12 gene activated the PI3K/Akt pathway in the brain tissues of anaesthetic-induced neuronal injury rats, thereby inhibiting apoptosis of neuronal cells. These data are supported by previous studies. Furthermore, the $\mathrm{PI} 3 \mathrm{~K} /$ Akt signalling pathway is activated by binding of BDNF to TrKB, thus decreasing apoptosis in neuronal cells. BDNF protects against neuronal cell death by decreasing apoptosis [1]. Inhibiting the P2Y12 gene reduced apoptosis of neuronal cells by enhancing expression of the BDNF protein.

\section{Conclusions}

In conclusion, our data revealed that inhibiting the P2Y12 gene with prasugrel ameliorated the neuronal injury induced by isoflurane in rats, by regulating the BDNF/TLR-4/TNF- $\alpha$ pathway. Inhibiting the P2Y12 gene could be a novel target for the development of therapeutic drugs for neuronal injury.

\section{Acknowledgements}

All authors of this manuscript thank Weifang People's Hospital for providing the facilities necessary for performing this study.

\section{Disclosure}

The authors report no conflict of interest.

\section{References}

1. Almeida RD, Manadas BJ, Melo CV, Gomes JR, Mendes CS, Grãos MM, Carvalho RF, Carvalho AP, Duarte CB. Neuroprotection by BDNF against glutamate-induced apoptotic cell death is mediated by ERK and PI3-kinase pathways. Cell Death Differ 2005; 12: 1329-1343.

2. Amadio S, Tramini G, Martorana A, Viscomi MT, Sancesario G, Bernardi G, Volonté C. Oligodendrocytes express P2Y12 metabotropic receptor in adult rat brain. Neuroscience 2006; 141: 1171-1180.

3. Barnhart CD, Yang D, Lein PJ. Using the Morris water maze to assess spatial learning and memory in weanling mice. PLoS One 2015; 10: e0124521.

4. Bathina S, Das UN. Brain-derived neurotrophic factor and its clinical implications. Arch Med Sci 2015; 11: 1164-1178.

5. Bovill JG, Sebel PS, Stanley TH. Opioid analgesics in anesthesia: with special reference to their use in cardiovascular anesthesia. Anesthesiology 1984; 61: 731-755.

6. Cheng Y, He L, Prasad V, Wang S, Levy RJ. Anesthesia-induced neuronal apoptosis in the developing retina: a window of opportunity. Anesth Analg 2015; 121: 1325-1335.

7. Chinn GA, Sasaki Russell JM, Sall JW. Is a short anesthetic exposure in children safe? Time will tell: a focused commentary of the GAS and PANDA trials. Ann Transl Med 2016; 4: 408.

8. Gachet C. P2Y(12) receptors in platelets and other hematopoietic and non-hematopoietic cells. Purinergic Signal 2012; 8: 609-619.

9. He J, Zhao J, Peng X, Shi X, Zong S, Zeng G. Molecular mechanism of MiR-136-5p targeting NF- $\mathrm{KB} / \mathrm{A} 20$ in the IL-17-mediated inflammatory response after spinal cord injury. Cell Physiol Biochem 2017; 44: 1224-1241.

10. Kalimuthu S, Se-Kwon K. Cell survival and apoptosis signaling as therapeutic target for cancer: marine bioactive compounds. Int J Mol Sci 2013; 14: 2334-2354.

11. Kim JY, Kim N, Yenari MA. Mechanisms and potential therapeutic applications of microglial activation after brain injury. CNS Neurosci Ther 2014; 21: 309-319. 
12. Kowiański P, Lietzau G, Czuba E, Waśkow M, Steliga A, Moryś J. BDNF: A key factor with multipotent impact on brain signaling and synaptic plasticity. Cell Mol Neurobiol 2017; 38: 579-593.

13. Lawrence T. The nuclear factor NF-kappaB pathway in inflammation. Cold Spring Harb Perspect Biol 2009; 1: a001651.

14. Lee JH, Zhang J, Wei L, Yu SP. Neurodevelopmental implications of the general anesthesia in neonate and infants. Exp Neurol 2015; 272: 50-60.

15. Liang G, Ward C, Peng J, Zhao Y, Huang B, Wei H. Isoflurane causes greater neurodegeneration than an equivalent exposure of sevoflurane in the developing brain of neonatal mice. Anesthesiology 2010; 112: 1325-1334.

16. Liu H, Dai T, Guo W. Isoflurane-induced neuronal apoptosis in developing hippocampal neurons. Neural Regen Res 2013; 8 825-832.

17. Liverani E, Kilpatrick LE, Tsygankov AY, Kunapuli SP. The role of $\mathrm{P}_{2} \mathrm{Y}_{12}$ receptor and activated platelets during inflammation. Curr Drug Targets 2014; 15: 720-728.

18. Li W, Li DY, Zhao SM, Zheng ZJ, Hu J, Li ZZ, Xiong SB. Rutin attenuates isoflurane-induced neuroapoptosis via modulating JNK and p38 MAPK pathways in the hippocampi of neonatal rats. Exp Ther Med 2017; 13: 2056-2064.

19. Schaar KL, Brenneman MM, Savitz SI. Functional assessments in the rodent stroke model. Exp Transl Stroke Med 2010; 2: 13.

20. Sun L. Early childhood general anaesthesia exposure and neurocognitive development. Br J Anaesth 2010; 105 (Suppl 1): i61-i68.

21. Vorhees CV, Williams MT. Morris water maze: procedures for assessing spatial and related forms of learning and memory. Nat Protoc 2006; 1: 848-858.

22. Wang S, Zhou Y. Baicalein inhibits neuroapoptosis via pathways in sevoflurane induced rats. Transl Neurosci 2018; 9: 88-98.

23. Wei H, Liang G, Yang $\mathrm{H}$. Isoflurane preconditioning inhibited isoflurane-induced neurotoxicity. Neurosci Lett 2007; 425: 59-62.

24. Wu B, Yu Z, You S, Zheng Y, Liu J, Gao Y, Lin H, Lian Q. Physiological disturbance may contribute to neurodegeneration induced by isoflurane or sevoflurane in 14 day old rats. PLoS One 2014 9: e84622.

25. Xi JS, Wang YF, Long XX, Ma Y. Mangiferin potentiates neuroprotection by isoflurane in neonatal hypoxic brain injury by reducing oxidative stress and activation of phosphatidylinositol-3-kinase/Akt/mammalian target of rapamycin (PI3K/Akt/mTOR) signaling. Med Sci Monit 2018; 24: 7459-7468.

26. Xu C, Shen G, Yuan X, Kim JH, Gopalkrishnan A, Keum YS, Nair S, Kong AN. ERK and JNK signaling pathways are involved in the regulation of activator protein 1 and cell death elicited by three isothiocyanates in human prostate cancer PC-3 cells. Carcinogenesis 2006; 27: 437-445.

27. Yamauchi K, Imai T, Shimazawa M, Iwama T, Hara H. Effects of ticagrelor in a mouse model of ischemic stroke. Sci Rep 2017; 7: 12088.

28. Zhang H, Zhang YW, Chen Y, Huang X, Zhou F, Wang W, Xian B, Zhang X, Masliah E, Chen O, Han JD, Bu G, Reed JC, Liao FF Chen YG, Xu H. Appoptosin is a novel pro-apoptotic protein and mediates cell death in neurodegeneration. J Neurosci 2012; 32 15565-15576. 